\title{
The Chemical Table: An Open Dialog between Visualization and Design
}

\author{
Francis T. Marchese \\ Department of Computer Science, Pace University, New York, NY 10038 \\ fmarchese@pace.edu
}

\begin{abstract}
With a history of over 300 years, the chemical table is the textbook case of information visualization. This paper explores the development of the chemical table as a tool designed for chemical information visualization. It uses a historical context to investigate the purpose of chemical tables and charts, and suggests reasons why the two-dimensional periodic table remains the de facto standard for chemical information display.
\end{abstract}

\section{Introduction}

The chemical table may be one of the earliest successful examples of information visualization. Created over 300 years ago as a tool to organize and visualize chemical reactivity, these tables have evolved into representations of chemical periodicity known today as periodic charts and tables. Moreover, during the past 150 years over seven hundred visualizations of chemical periodicity have appeared, including fans, two and threedimensional spirals and loops, as well as a diverse array of two-dimensional tabular forms. The sheer number of visual depictions not only points to the importance played by chemical tables and charts as organizing constructs for the fundamental components of the chemical knowledge but also the continued search for a complete, coherent, systematic representation of the organization of chemical information. Yet, despite the sea of charts that has been devised to date, the twodimensional periodic table remains the de facto representation that not only defines the chemical information domain, but also is used as an icon for the organization of information in general.

Chemical tables and charts are visual representations of chemical information composed of data such as atomic weights, ionization potentials, oxidation states, reaction rates, melting points, crystal structures, and alike. Although these are experimentally measurable quantities, many of which represent complex atomic or molecular processes, they have neither intrinsic nor causal geometric correlations such as those found in the three-dimensional arrangement and bonding patterns of atoms within molecules. Hence, a table or chart of the melting points of the chemical elements must be considered a visualization of abstract data organized on a conceptual substrate such as a $x y$ grid.

Chemical tables are designed artifacts. They are created to organize chemical knowledge, and used as tools in the reasoning about it. They are dynamic as well. Their structure has been changed and adapted over time to accommodate new chemical data and chemists' perceptions on how to better organize it. As Cohen has observed: "tables are components of and contributors to the material culture of chemistry" and "share a legacy of visual practices" [1] integral to chemical research and education.

The purpose of this paper is to review the chemical table from an historical perspective as a designed tool for information visualization whose form and use have changed over time based on chemists' evolving understandings of chemical knowledge. This paper may be viewed as an open-ended case study that communicates a sequence of snapshots of the on-going process of information visualization in chemistry. The following sections cover chemical information expressed as tables, charts, and models. The paper concludes with a comparison and analysis. In particular, it emphasizes the process of periodic table design, and suggests why the two dimensional periodic table remains the standard design. For those interested in the early history of chemical tables see Cohen's review [1]. Scerri [2] and van Spronson [3] review the history of the periodic table. Mazurs [4] organizes and catalogs the myriad of representations. An on-line tabulation of periodic tables with references is found at the Meta-Synthesis website [5]; while two of the many interactive periodic tables may be viewed at WebElements [6] and as a Wikipedia entry [7].

\section{Tables}

\subsection{Early Tables}

One of the earliest chemical tables was published in 1718 by Étiénne-François Geoffroy [8]. The "Table des différents rapports observés en Chimie entre différentes substances" (Figure 1) was designed to systematically organize the rapidly accumulating chemical reaction data 
and to be used as a guide to laboratory practice. The table of chemical affinities is organized as a grid, displaying cells that contain symbols representing the chemical substances defined in the table's legend. At the head of each vertical column is the symbol for a specific chemical substance; below it are its reactive partners, arranged in decreasing order of degree of reactivity.

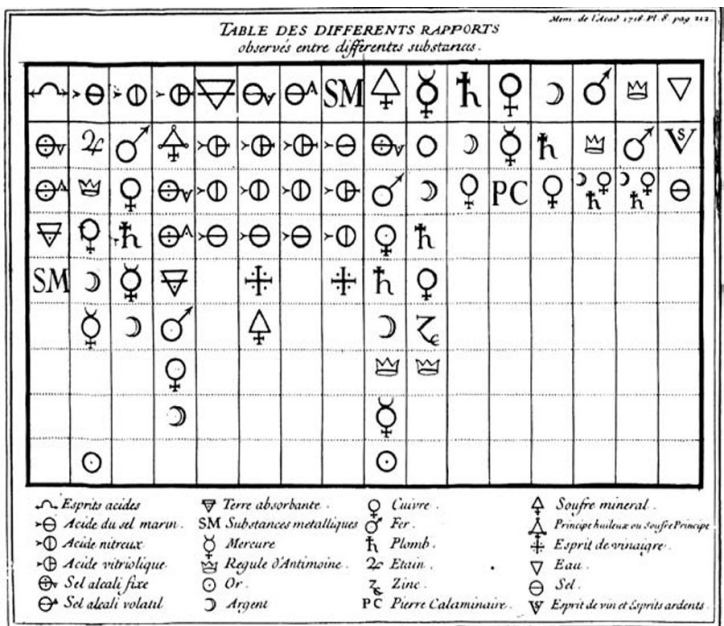

Figure1 Étiénne-François Geoffroy’s “Table des différents rapports.

Geoffroy designed the table as a tool in order to show a chemist how to go about selecting specific chemical procedures. For example, if a chemist wanted to separate silver from a nitrous acid silver mixture he could look at column three of table to find a substance that possessed a greater affinity for nitrous acid. Here he would find that either lead $(\hbar)$ or iron $\left(\delta^{\Uparrow}\right)$ would react better with the acid and displace silver (O) because each held a higher position in the column.

What is notable about Geoffroy's table is the large number of empty cells - places where future affinities should reside. Geoffroy commented that this was a design decision with the expectation that future research would fill in the blanks. His agenda is clearly evident in column two of the table where the last cell is occupied by gold $(\odot)$, leaving two empty cells between it and mercury $(\%)$.

The usefulness of Geoffroy's table found its momentum in the latter quarter of the eighteenth century with chemists adding new columns for new substances. The culmination was a 59 column by 50 row table published by Torbern Bergman in 1783 (c.f. [1]). Compared to Geoffroy's single page document of sixteen columns, Bergman's multi-page table was unwieldy and difficult to use. Bergman's table was an example where completeness trumped usefulness; indicative of a design that did not scale or evolve to accommodate the greater complexity of information.

\subsection{Rise of Mendeleev's Table}

Designs for periodic tables of the elements began in the early nineteenth century, when the availability of reasonably accurate atomic weights made it possible for chemists to postulate the possible patterns of relationships that were expected to exist among the chemical elements. Doing chemistry at that time in history meant experimentation and classification, the ultimate goal of which was to discover Nature's designs the underlying schemes that govern the structure, function and development of chemical systems. In effect, the design of a chemical table should reflect the natural organization of the elements.

Beginning with William Prout's 1815 hypothesis that all chemical elements possessed atomic weights that were whole number multiples of hydrogen, chemists attempted to order elemental properties based on atomic weight. Such data was employed in numerological experiments in which atomic or equivalent weights were averaged, ratioed, or algebraically related. A periodic table published by John Newland's in 1863 was based on his Law of Octaves, that likened the repetition in atomic property for every eighth element in his table to the eighth note in the musical octave. This table was much ridiculed because of its metaphorical underpinning; yet in retrospect, it did order many of elements correctly.

The watershed event in periodic table design came when Dimitri Mendeleev reported his periodic table in a paper presented to the Russian Chemical Society on March 6, 1869 (Figure 2) [9]. Instead of taking an algebraic approach, Mendeleev developed his design by arranging sixty-three index cards, each representing a known element, onto which he had written a collection of known physical properties. He set out to find their associations by placing each card on a table, row by row, according to increasing atomic mass. A new row was begun whenever an element exhibited physical properties similar to the element above it on the table. Conversely, positions were left empty if an element's properties did not match with neighbors. In the end, Mendeleev arrived at the table shown in Figure 2.

Mendeleev's table is important for both what it does and does not show. First, elements with common properties are arranged along rows into families (e.g. Li, $\mathrm{Na}, \mathrm{K}, \mathrm{Rb}, \mathrm{Cs}$, and $\mathrm{Tl}$ ). Second, empty positions in his table (denoted by question marks, which include estimated atomic weights), are places where elements would be expected to occupy if they had been discovered. In the latter case Mendeleev used his periodic table as a foundational theory to predict in detail the properties of the missing elements that were later to be called scandium, gallium, technetium, and germanium. This use of the periodic table as a predictive tool is what sets apart Mendeleev's table from other representations of his time, and garners him the prestige of being labeled the father of the periodic law.

It should be noted that Mendeleev's first table was far from his last. He constantly tinkered with its organization. Bensaude-Vincent has observed [10] that 
over the course of eight editions of his textbook Principle of Chemistry, he changed his table many times - displaying five variations within the sixth edition alone! Clearly, he did not favor any one representation, because none were completely satisfactory. Yet, the periodic table's plasticity provoked tinkering as part of his chemical reasoning process. This point will gain emphasis further along into this paper as more tables are considered.

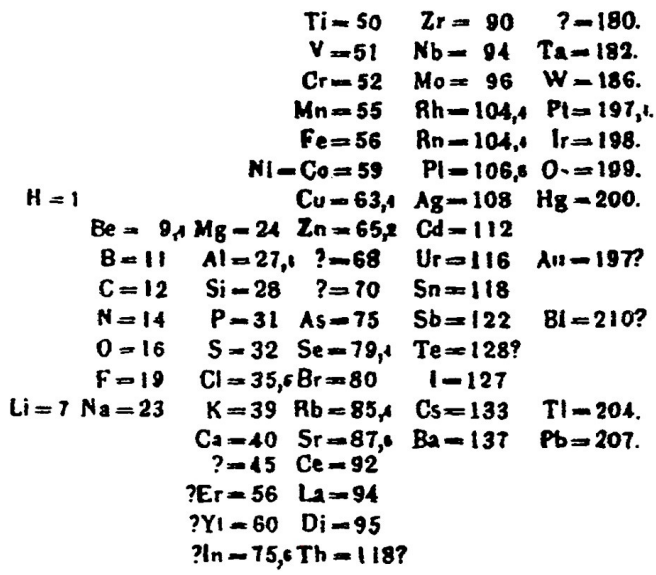

Figure 2 Mendeleev's periodic table of 1869.

\subsection{Contemporary Tables}

Experiments in atomic physics at the end of the nineteenth century and development of quantum theory during the first two decades of the twentieth century provided new ways of assessing and representing the interrelationships among the chemical elements. In particular, the use of atomic numbers (i.e. the number of protons in an atom's nucleus), and an element's electron configuration, made systematic arrangements of the elements more precise, but not necessarily more accurate.

For example, the left-step periodic table of Charles Janet (1927) [11] arranges the elements by how the outer electron shell of each element is filled (Figure 3). The chart is systematic, logical, and reflects the physical structure of each element at the atomic level. From leftto-right, the elements form blocks based on the electron configurations $-f$ to $d$ to $p$ to $s$ electrons. The table is read by beginning with the top right columns, moving across from $\mathrm{H}$ to $\mathrm{He}$ to fill the $1 s$ electron shell. Then the reader drops to the second row, moving from $\mathrm{Li}$ to $\mathrm{Be}$ to fill the $2 s$ shell. Moving across row three fills the $2 p$ and $3 s$ shells; and so forth.

For a physicist who is interested in atomic structure, this may be a perfectly fine arrangement, but for a chemist who is concerned with the properties of substances (i.e. collections of atoms), there are problems with this design. In particular, Helium (He), a noble gas, is situated at the far right of the table with the alkaline earths (Be, Mg, etc.), when it should be aligned with the other elements of the noble gas family ( $\mathrm{Ne}, \mathrm{Ar}, \mathrm{Kr}$ ).

This problem points to the issue of point-of-view in the use of the periodic table. Chemists require the periodic table's design to be consistent with their perceptions of how the elements organize into groups based on an element's chemical properties. But because much of chemistry is reducible to physics, in particular quantum theory, the periodic table's design should reflect in some way its theoretical physical foundations.

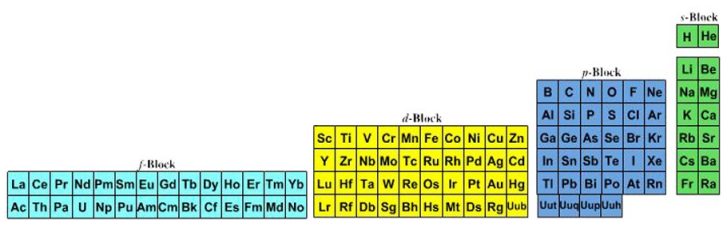

Figure 3 Janet's periodic table of 1927.

Today's standard periodic table is a variation of Janet's proposed design, reflecting chemists' requirement of natural chemical grouping, given the constraints of atomic physics. In the contemporary table exemplified by the United States National Institute of Standards and Technology (NIST) Periodic Table shown in Figure 4 [12], all of the $s$-block elements except for helium are moved to the left-hand side; helium is placed above the noble gases. The electron shells are still filled in a logical manner, consistent with experiment. For the sake of graphical layout, the $f$-block elements consisting of the lanthanides and actinides are placed below the main group. This arrangement makes the $f$-block appear as an independent entity, as seen in many renderings, but the NIST table maintains the connection with the main table by using a ribbon. This overall graphical design is important to making the contemporary table a usable tool because it can easily fit on letter or A4 size pages, in textbooks, and now web pages.



Figure 4 Contemporary Periodic Table by NIST.

\subsection{Other Designs}

The contemporary table is by no means the last word in periodic table designs. Since it represents a 
compromise between usability and an ideal, it continues to stimulate rearrangement and adaptation. Two such examples are show in Figures 5 and 6 . The first is a pyramidal table originally devised by Thomas Bayley in 1882 [13], revised by Jørgen Thomsen in 1895 [14], Neils Bohr in 1922 [15], and recently revised yet again by William Jensen in 1989 [16]. It is a long table designed to show the symmetry of the periodic law as it relates to each period. Finally the periodic spiral by Electric Prism [17] breaks with the enforced regimentation of the $x y$ grid, embedding the elements in a hexagonal lattice. Designed by Jeff Moran, it attempts to address the issue of where to place hydrogen in the table. The chemistry of hydrogen suggests it could be placed either above lithium or fluorine in the contemporary table. Moran's table solves this problem by making hydrogen a neighbor of both. It is situated next to helium as well, showing that hydrogen precedes helium in the filling of electron configurations.

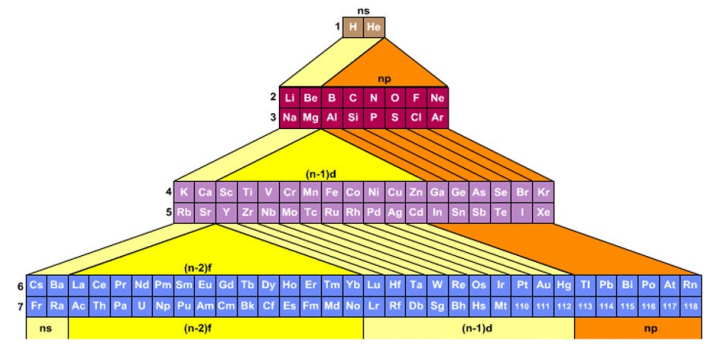

Figure 5 Bayley-Thomsen-Bohr Periodic Table.

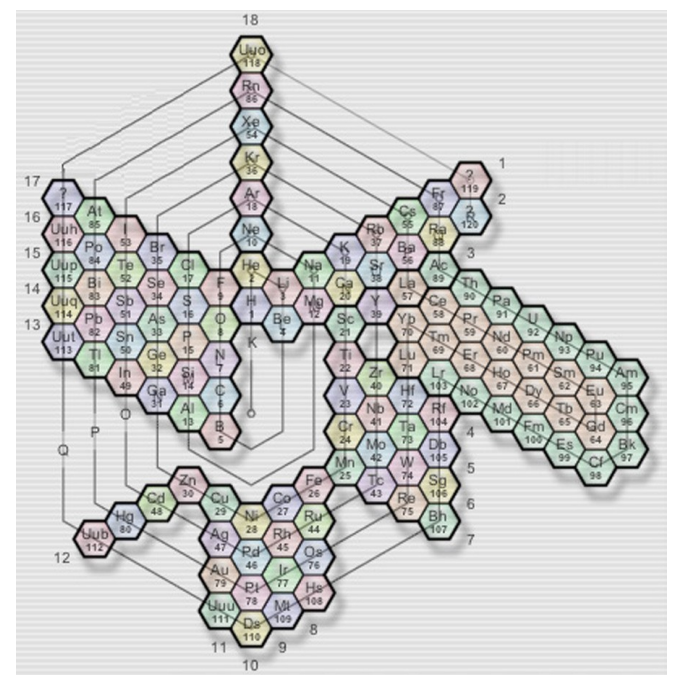

Figure 6 Periodic Spiral by Electric Prism, Inc.

\section{Charts and Physical Models}

\subsection{Chemical Charts}

The earliest use of a chart to demonstrate the periodic nature of the elements was Alexandre-Émile Béguyer de Chancourtois's Telluric Screw of 1862 [18]. De Chancourtois proposed a three-dimensional helical representation, where elements spiraled around a cylinder (Figure 7). This is a remarkable proposal, considering that few three-dimensional data visualizations existed through the first two-thirds of the nineteenth century. Somehow de Chancourtois made the leap beyond the $x y$ plane, not just into the orthogonal $z$ direction, but into a new reference frame - cylindrical coordinates. Where would he get an idea for such a construction?

It is possible that de Chancourtois's charting practice was inspired by a recording device of the time - the cylindrical drum and stylus. Because de Chancourtois was interested in detailed classifications of phenomena such as earthquakes, ground waves, and oscillations, he was familiar with these recording devices, particularly as they applied to seismology. A method of chart recording invented by Thomas Young, and advanced by Charles Wheatstone and Abraham-Louis Breguet had existed since 1807, used a drum rotated by clockwork. As the drum rotated on its axis, it descended past a fixed stylus. For every $360^{\circ}$ turn, the drum sank a quarter inch. The net result was a smooth spiral traced across the drum's surface.

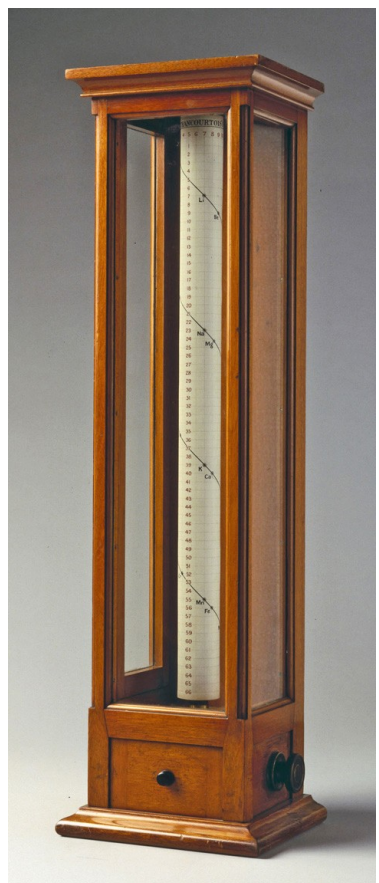

\section{Figure 7 de Chancourtois's Telluric Screw. CScience Museum, London. Used with permission.}

It is proposed here that de Chancourtois utilized the stylus/drum metaphor to devise a paper tool that would allow him to explore the periodic relations among the elements. He first created a plot of atomic weights along the $45^{\circ}$ diagonal of a sheet of graph paper. De Chancourtois could then roll the paper into a cylindrical spiral. The spiral would be tightened or relaxed until elements exhibiting similar properties were in vertical 
alignment. He found that by aligning elements in multiples of 16 weight units, he arrived at a period repeat of 16. This alignment correctly creates families of elements such as oxygen, sulfur, selenium, and tellurium; and lithium, sodium, and potassium.

A spiral/radial alignment chart was published by Gustavus Detlef Hinrichs in 1867 [19], predating Mendeleev's table by two years. A relatively recent spiral chart designed by Theodor Benfey's in 1960 is shown in Figure 8 [20]. In the color encoded version illustrated here, the elemental families radiate out from the center of a clockwise spiral with hydrogen at its origin.

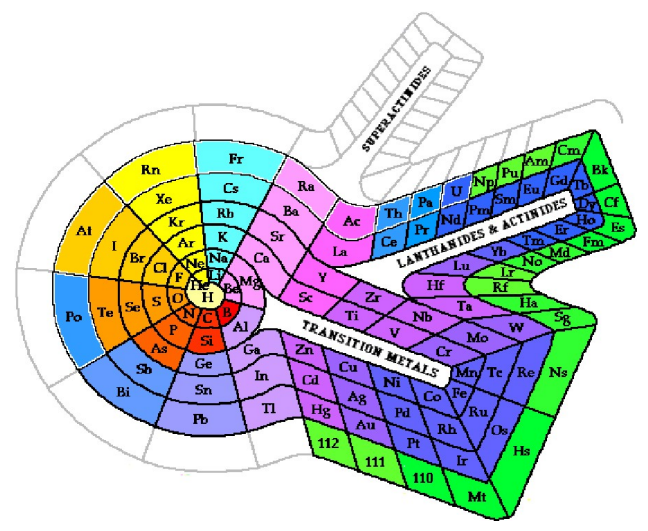

Figure 8 Benfey's spiral table of 1960.

These kinds of charts may be quite complex as well. Figure 9 displays a chart created by Georg Schaltenbrand in 1920 [21]. It is composed of two parts, each showing a different view of helices of varying sizes consisting of 2 , 6,10 , and 14 elements. The top segment of the chart is a perspective projection of the three dimensional helices viewed on side, and the bottom segment contains a parallel projection looking down through the structure. Mazurs has commented that this chart "must be considered to be the best table for presenting the periodic law in all its details," [4] because each $360^{\circ}$ turn of the helix clearly shows each chemical period. But from a design viewpoint, it is undoubtedly both difficult to construct and to read. Moreover, the three dimensionality of the helices would be better served by creating a three dimensional physical model.

\subsection{Physical Models}

A few three dimensional physical models of the periodic system have been built. Physical models are attractive from a design perspective because they occupy the three-dimensional space of the user and provide tactile feedback. They can be oriented and reoriented, measured, assembled, disassembled, and rearranged by hand. The most significant problem facing model builders is the model's complexity. A model of Schaltenbrand's helices would be difficult and time consuming to build.

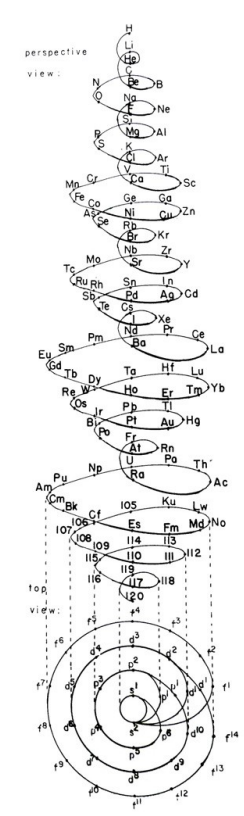

Figure 9 Schaltenbrand's helices of 1920.

Two examples of physical models will be presented. The first considered is an early physical model constructed by William Crookes in 1888 [22] called the pretzel model. Figure 10 shows his model which currently resides in the collections of the London Science Museum. Crookes designed the pretzel model to illustrate the sequence in which the elements were created during the evolution of the universe (e.g. H, He, $\mathrm{Li}$, etc.). It was meant to be an improved diagrammatic tool for depicting the periodic table by extending his two dimensional $x y$ periodic plots into the third dimension. As with Mendeleev, Crookes' design also contained positions for future elements. But from a chemist's perspective, this model's design was poor because all periods were of the same length.

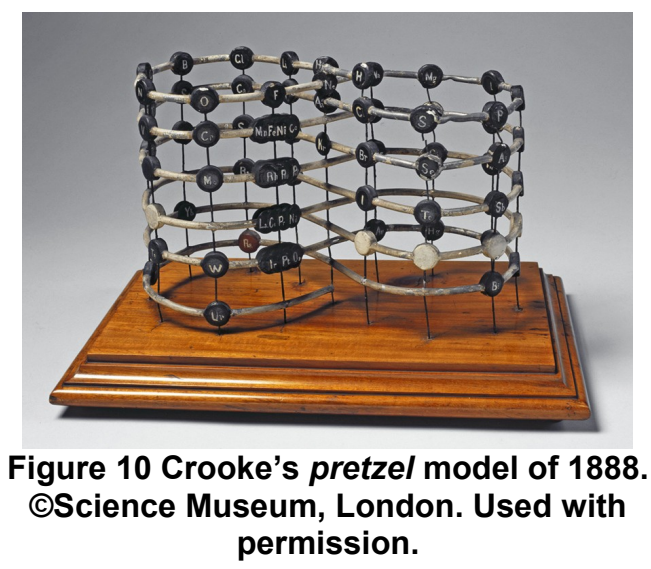

The second model was built by Roy Alexander (Figure 11) [23], whose design advances the cardboard model constructed by Courtines [24] and Clark [25] in 1925 , by accurately integrating the actinides and lanthanides into the table. Alexander's model is an 
excellent educational tool. The student version is supplied as a collection of die cut sheets of cardboard that must be colored and assembled. This makes modeling of the periodic table a participatory process.

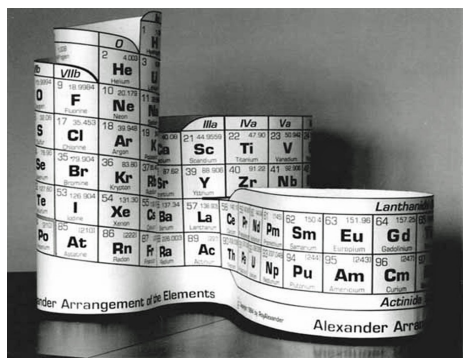

Figure 11 Alexander's periodic model.

\section{Analysis}

At the beginning of this paper it was stipulated that the two-dimensional periodic table remains the de facto representation of chemical information. Clearly, complex three dimensional charts and physical models covered here (and those compiled by Mazurs [4]) are difficult to construct, and even more difficult to interpret without machine intervention. Yet, the advances in computer graphics and interface technology of the past four decades have not been employed to build systems that display these complex relationships. Instead, these technologies have been used to transform the way chemists view and interact with three dimensional molecular structure and dynamics. In contrast, chemists have capitalized on web technologies to construct interactive periodic tables exploiting its contemporary design as a user interface to give access to a wealth of chemical information (e.g., WebElements Scholar organizes sixty different elemental properties). This point is of particular note. Since chemists themselves are the primary builders of their own visualization systems [26], they could have synthesized interactive threedimensional periodic charting software. Evidently, two dimensional tables fulfill a richer set of design requirements.

\subsection{Table Usage}

It is well understood that tables are important data visualization tools and the first stage in the information visualization pipeline that organizes raw data into a form that may translated into graphics such as the periodic charts presented in Figures 8 and 9. Here, though, the table is the visualization itself, each cell of which contains chemical iconography (e.g. the alchemical symbols found in Geoffroy's table (Figure 1) or the standard chemical symbols used in periodic tables (Figures 2 and 4)).

The power of the table as a visualization medium is that it is a compactly organized structure that facilitates understanding of relationships among different data elements within it. In an analysis of tables, Wainer [27] [28] has put forward four rationales for table usage: exploration, communication, storage, and illustration. In exploration, tables help answer questions about the data. In communication, tables provide effective means for presenting data - each table has a story or stories to tell. Storage archives data, supporting a historical context and aiding in data retrieval. In illustration, tables are used as graphics in support of narrative.

Wainer's rationales may be employed to analyze chemical tables in general. Here Geoffroy's table (Figure 1) is utilized as a specific example. Geoffroy's table was designed to answer questions about reactivity. Chemists explored his table to find the best displacement reaction to solve their laboratory separation problems. It was an efficient communication medium as well, because it translated the chemical narrative detailing the systematics of chemical reaction selection into an easily understandable graphic form. Geoffroy's table was an open-ended storage medium containing a history of chemical practice that could be appended to over time. His table design functioned so well as a tool that its size ballooned into Bergman's behemoth of 1783. Finally, Geoffroy's table is an illustration that serves the greater narrative of chemical synthesis, acting as a focal point for analysis and discourse.

\subsection{Table Design}

A table's design must reflect the kinds of questions that will be asked of the data it contains [26][27]. There are three levels of inquiry.

First, elementary questions about data involve single category data inspection or extraction. In a modern periodic table (c.f. Figure 4) each location is a box, a container that displays atomic data. Electron configurations, atomic numbers and weights, crystal structures, freezing and boiling points are all tossed into the bin. This is in direct contrast with a chemical chart that represents elements as points in space (e.g. Figure 9). Hence, the advantage of the table over the chart is that points are merely placeholders, while boxes are containers with spatial extents that encapsulate collections of data.

Second, intermediate questions about data involve comparisons, trends, and relationships among data entries. Chemical tables were designed for this purpose to organize chemical information so the relationships among chemical properties may be exposed. Indeed, since each element in the contemporary table is represented by a geometric primitive (typically a rectangle), its graphic attribute may be adjusted to highlight trends across families of elements or to pinpoint elemental outliers. Color is used widely, but other primitives have been employed. For example, scaled circles displayed at table locations are used to convey relative atomic size. Overall, chemical tables are coherent graphical entities consistent with Bertin's rules for visual encoding [29]. 
Finally, general questions about data require a thorough understanding of the underlying data structure and the ability to build a level of abstraction for the entire data set. The chemical table's continued design and redesign demonstrates chemists' evolving understanding of the underlying conceptual abstractions that define the nature of chemical elements and their associations.

\subsection{Tool Design}

The chemical table carries the added responsibility of being a research and teaching tool. Some requirements for a well designed visualization tool include: direct access to data, accurate encoding of information, easy integration into work practice, and short learning curve. Two dimensional chemical tables meet these requirements - charts and models do not. Moreover, interactive chemical tables implemented in hypermedia technology fulfill a further set of requirements including: the ability to readily display meaningful patterns, trends, and exceptions; the use of visualization best practices; and the dynamic linking of table contents to supporting chemical documentation and narrative.

\section{Conclusion}

This paper has explored the development of the chemical table as a tool designed for chemical information visualization. By examining tables in general, it has shown why the design of the twodimensional periodic table remains the de facto standard for chemical information display. Finally, considering the practice of chemical table and chart designers, this paper has emphasized the fact that the periodic table is not a static iconic representation, rather it, and each box contained within it, is dynamic, the design and content of continues to change with chemists' needs.

\section{References}

[1] B.R. Cohen, "The element of the table: Visual discourse and the preperiodic representation of chemical classification," Configurations 12, 1, Winter 2004, pp. 41-75.

[2] E.R Scerri, The Periodic Table: Its Story and Its Significance, Oxford, Oxford University Press. 2007.

[3] J.W. van Spronson, The Periodic System of the Chemical Elements: A History of the First Hundred Years, Amsterdam, Elsevier, 1967.

[4] E.G. Mazurs, Graphic Representations of the Periodic System During One Hundred Years, $2^{\text {nd }}$ Edition, University of Alabama Press. 1974.

[5] Meta-Synthesis, http://www.metasynthesis.com/ webbook.html, accessed March 14, 2008.

[6] WebElements, http://www.webelements.com/, accessed March 14, 2008.

[7] Wikipedia Periodic Table, http://en.wikipedia.org/ wiki/Periodic_table, accessed March 14, 2008.

[8] É-F Geoffroy, "Table des differents rapports observés en chimie entre differentes substances," Mem. Acad. R. Soc. 1717, pp. $202-212$.
[9] D. Mendeleev, "Sootnoshenie svoistv s atomnym vesom elementov," Zh. Russk. Khim. Obshch. 1, 1869, pp. 60-77.

[10] B. Bensaude-Vincent, "Graphic representation of the periodic system of the elements." In Tools and Modes of Representation in the Laboratory Sciences edited by U. Klein, Kluwer Academic Publisher, Dordrecht, The Netherlands, 2001, pp. 133-161.

[11] C. Janet, "La structure du noyau de l'atome, considerde dans la classification periodique des elements chimques," Beavis, Novenbre, 1927.

[12] NIST Periodic Table, http://physics.nist.gov/ PhysRefData/PerTable/index.html, accessed March 14, 2008.

[13] T. Bayley, "On the connection between the atomic weight and the chemical and physical properties of the elements," Phil. Mag. 13, 1882, pp. 26-37.

[14] J. Thomsen, "Systemachische gruppierung de chemischen elemente," Z. Anorg. Chem. 9, 1895. pp. 190-193.

[15] N. Bohr, "Drei aufsätz über spektren und atombanu." Braunschweis: F. Viewes \& Sohn, 1922.

[16] W.B. Jensen, "Classification, Symmetry, and the Periodic Table," Computing and Mathematics with Applications 12B, 102, 1989, pp. 487 -510.

[17] Electric Prism Periodic Spiral, http://www.periodicspiral.com/, accessed March 14, 2008.

[18] A.E. Beguyer de Chancourtois, "Vis tellurique: classment naturel des coups simples ou radicaux obtenu au moyen d'un systeme de classification helicoidal et numerique," Paris. 1863.

[19] G.D. Hinrichs, Programm der Atomechanik, oder die Chemie eine Mechanik der Panatome. Iowa City, 1867, pp.7-8.

[20] T. Benfey, http://homepage.mac.com/ dtrapp/periodic.f/spiral.html, accessed March 14, 2008.

[21] G. Schaltenbrand., "Darstellung des periodic des periodischen systems der elemente durch eine raumliche spirale. Z. Anorg. Allgem. Chem. 112, 1920, pp. 221-224.

[22] W. Crookes, "On the position of helium, argon, and krypton in the scheme of the elements," Proc. Roy. Soc. (London) 63, 1898, pp. $408-411$.

[23] R. Alexander, "Periodic Tables," http://allperiodictables.com/ClientPages/AAEpages/apt_ 3_DeskTopper.html, accessed March 14, 2008.

[24] M. Courtines, J. Chem. Educ. 2, 1921, p. 107.

[25] J.D. Clark, J. Chem. Educ. 10, 1921, p. 675.

[26] F.T. Marchese. "Molecular visualization at the interface." In Trends in Interactive Visualisation / Advanced Information and Knowledge Processing Series edited by Elena Zudilova-Seinstra, Tony Adriaasen and Robert van Liere, Springer-Verlag, 2008. in press.

[27] H. Wainer, "Understanding graphs and tables," Educational Researcher, 21, 1, January- February 1992, pp. 12-23.

[28] H. Wainer, "Improving tabular displays, with NAEP: Tables as examples and inspirations," Journal of Educational and Behavioral Statistics, Spring 1997, 22, 1, pp. 1-30.

[29] J. Bertin, Semiology of Graphics (translated by W.J. Berg), University of Wisconsin Press, 1983. 